\title{
Giant seborrheic keratosis of the face - An unusual presentation
}

\author{
Koh Khai Luen ${ }^{1 *}$, Rashid Shawaltul Akhma ${ }^{2}$, Wan Sulaiman Wan Azman ${ }^{1}$ \\ ${ }^{1}$ Reconstructive Sciences Department, Hospital Universiti Sains Malaysia, Health Campus, Universiti Sains Malaysia, 16150 Kubang Kerian, \\ Kota Bharu, Kelantan, Malaysia \\ ${ }^{2}$ Department of Plastic and Reconstructive Surgery, Hospital Raja Perempuan Zainab II, 16150 Kubang Kerian, Kota Bharu, Kelantan, Malaysia
}

\begin{abstract}
Seborrheic keratosis is the most common benign epidermal lesion in the world, especially among the elderly. Its inherent benign nature has precluded the need to remove it for medical reasons. Most of the concerns presented to dermatologists or plastic surgeons are of cosmetic reasons, besides some unusual appearances that necessitate cutaneous malignancy evaluation. Unusually large sizes of seborrheic keratosis are rarely reported, and its clinical significance is largely unknown. It has been proven by recent molecular studies that seborrheic keratosis is true neoplasia rather than a mere epidermal hyperplasia, and various authors have reported several cases of concomitant malignancy arising from seborrheic keratosis. Plastic surgeon expertise is often required when faced with an extensive lesion, requiring reconstructive procedures to preserve good aesthetic and functional outcomes. The purpose of this review is to report a case of an unusually large seborrheic keratosis on the face, highlighting its clinical relevance and surgical management.
\end{abstract}

Keywords: face; giant seborrheic keratosis; surgical management

Citation: Koh KL, Akhma RS, Wan Azman WS. Giant seborrheic keratosis of the face - An unusual presentation. J Dermatol 2016; 1(1): 25-28; http://dx.doi.org/10.18282/jsd.v1.i1.34.

*Correspondence to: Koh Khai Luen, Reconstructive Sciences Unit, Universiti Sains Malaysia, Health Campus, 16150 Kubang Kerian, Kelantan, Malaysia, kkhailuen@ hotmail.com.

Received: $13^{\text {th }}$ January 2016; Accepted: $11^{\text {th }}$ February 2016; Published Online: $21^{\text {st }}$ April 2016

\section{Introduction}

Seborrheic keratosis is one of the most common benign epidermal cutaneous lesions encountered by dermatologists and plastic surgeons in their daily practice ${ }^{[1,2]}$. However, given its benign nature, removal for medical reasons are unnecessary unless a histological confirmation of the clinical diagnosis is required, or the lesion has become traumatised and symptomatic. The most common concern for patients is often on the cosmetic outcome, especially when the lesion is on the face. Some unusual appearances of the lesions, such as gradual increase in size and pigmentation, may also serve as a concern for patients to present themselves to dermatologists for cutaneous malignancy evaluation ${ }^{[2]}$.
Seborrheic keratosis was first described in 1869 by Neuman as 'senile warts' since it commonly affected the elderly population, with a verrucous-like 'stuck on' appearance $^{[3]}$. Bathelemy was the first to coin the term 'seborrheic warts' in $1896^{[3]}$. It can occur anywhere on the body, but more commonly on sun-exposed areas of the face, trunk and extremities except the palm and sole. Historically, the lesions were merely thought to be epidermal hyperplasia until the advancement in molecular study emerged. A clonality analysis had shown that the majority of lesions are monoclonal tumours and have true autonomous growth, but without chromosomal instability. The growth of the epidermal cells are arrested at the G1 phase, hence maintaining its biologically benign nature ${ }^{[1]}$. Various authors had reported a concomi-

Copyright $@ 2016$ Koh KL, et al. This is an Open Access article distributed under the terms of the Creative Commons Attribution-Non Commercial 4.0 International License (http://creativecommons.org/licenses/by-nc/4.0/), permitting all non-commercial use, distribution, and reproduction in any medium, provided the original work is properly cited. 
tant occurrence of malignant growth associated with seborrheic keratosis, although without strong association of it ${ }^{[4-9]}$.

Unusually large-sized seborrheic keratoses are rarely reported, and their clinical significance is largely unknown. The majority of lesions occur in multiplicity with sizes less than $3 \mathrm{~cm}$, and they can usually be removed with non-surgical options ${ }^{[1]}$. Baer emphasised that the lesions can become very large and he published a case of giant seborrheic keratosis with the size of $5.5 \times 3.5 \mathrm{~cm}$ at the inguinal region ${ }^{[3]}$. Other authors had described the rarity of this tumour at the perigenital area with the largest dimension of $15 \times 10 \mathrm{~cm}$, warranting excision and reconstruction $^{[10]}$. The aim of this review was to report an unusually large, seborrheic keratosis on the face, by highlighting its clinical relevance and surgical management.

\section{Materials and methods}

The patient was a 75-year-old male with no known medical illnesses who had a large, painless, pedunculated lesion on the right side of his face for the past 30 years. In addition, there were similar multiple, yet smaller swellings around the face, scalp and neck. The patient came to seek treatment due to the growing mass pulling down on the upper right eyelid, and it has affected his vision on the right lateral gaze (Figure 1A, 1B). There was no significant history of bleeding from the mass, nor any infestation or infection. The history of skin exposure to sunlight was elicited.

Clinically, there was a $20 \times 15 \mathrm{~cm}$ oval-shaped pedunculated lesion, which appeared with an uneven surface and verrucous-like at the right temple, pulling down the right lateral upper eyelid. It was firm in consistency, mobile and with a well-demarcated stalk at the base (Figure 1C). There were also multiple, various-sized flat lesions stuck on the forehead, scalp and cheek. There were no clinically palpable neck nodes. The visual field at the right lateral gaze was reduced.

\section{Results}

A clinical diagnosis of giant seborrheic keratosis was made and a surgical excision was performed. The defected area was closed with a simple advancement flap from the surrounding skin laxity (Figure 1D). Figure 1E depicts gross appearance of the lesion. Histopathological examination (HPE) showed hyperkeratotic subtype of seborrheic keratosis without cellular atypia. After a 6-month follow-up, the scar was inconspicuous with an improvement in the function of the right eyelid, and there were no recurrences (Figure 1F). The patient has opted for conservative management of other lesions.
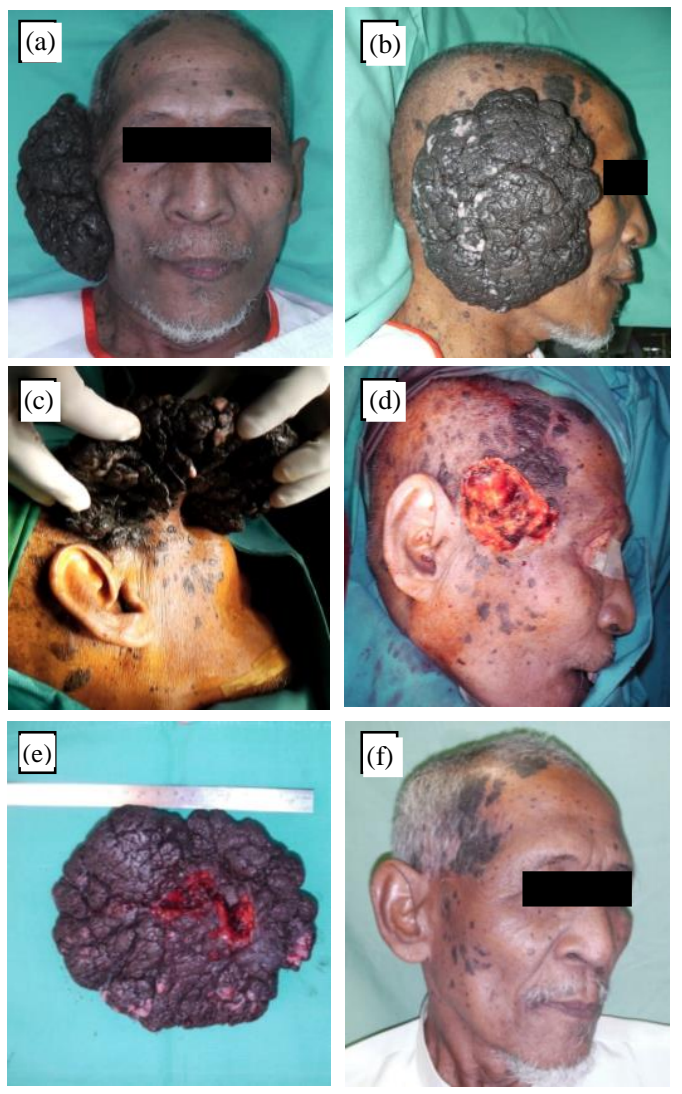

Figure 1. (A, B) Preoperative photo showed extension of the lesion and distortion of the right upper eyelid due to the gravitational effect of the mass (C) Intraoperatively, the tumour was pedunculated with stalk arising from the right temple region (D) Incision made along the base of the lesion leaving a defect size of $5 \times 6 \mathrm{~cm}$ (E) Gross appearance of the lesion (F) Postoperative photo after 6 months showed inconspicuous scar and normal right upper eyelid

\section{Discussion}

Seborrheic keratosis is readily diagnosed clinically, especially among the elderly, with its classical appearance. However, not all verrucous lesions are seborrheic keratosis $^{[11]}$. Clinical dilemma arises in flat-like lesions which have a smooth surface and pigmentation, or unusually large lesions mimicking malignant growth, especially at sun-exposed areas. Lesions need to be differentiated from other cutaneous malignancy. Thomas et al. reported a case of clinically typical seborrheic keratosis, but histologically proven to be malignant melanoma ${ }^{[12]}$, while Tsai et al. reported a case of sebaceous carcinoma, which was found to be contagious with seborrheic keratosis of 
the abdomen ${ }^{[8]}$. All suspicious lesions with diagnostic dilemmas or atypical presentations always require surgical excision and histological confirmation.

Despite the benign nature of seborrheic keratosis, various authors have reported the concomitant cases of malignant skin tumours associated within these lesions ${ }^{[5,7,12-14]}$. This may represent a probability of tumour collision rather than malignant transformation of the seborrheic keratosis; however, it cannot be excluded entirely. Bowen's disease is the most common neoplasm (7\%) followed by basal cell carcinoma (4\%), and up to $10 \%$ of excised lesions have a certain degree of atypia ${ }^{[6]}$. Lim, in 2006, retrospectively reviewed 639 consecutive histologically diagnosed seborrheic keratosis and found that 44 cases $(7 \%)$ have associated lesions which were malignant lesions, with 4 cases arising from seborrheic keratosis $^{[15]}$. This study was quickly followed by Vun $e t$ al. where they published their review of 813 cases of seborrheic keratosis, in which 45 cases were associated with non-melanoma cancer which were mainly basal cell carcinoma ${ }^{[16]}$. Both studies concluded that the predominant site for such malignant findings were on the face, especially at the temple region, but did not specifically mention the characteristics on seborrheic lesions that were associated with malignancy. Hence, it should be emphasised that seborrheic keratosis at sun-exposed areas is at a higher risk of concomitant malignancy, and histopathological diagnosis is warranted despite its benign nature. In our case, the lesion arose from the right temple region but lacked typical malignant changes besides its unusual size.

Seborrheic keratosis is also associated with other clinically important variants: namely stucco keratosis, dermatosis papulose nigra and Leser Trélat syndrome ${ }^{[17]}$. Leser Trélat syndrome warrants special consideration as it is a paraneoplastic syndrome with abrupt eruptions of numerous seborrheic keratosis ${ }^{[18]}$. It is often associated with internal malignancy such as adenocarcinoma. Molecular study has shown an association of PIK3CA gene mutation involved in the pathogenesis of seborrheic keratosis. This gene poses oncogenic properties in a number of malignant tumours such as the colon, breast and bladder cancers. Hence, seborrheic keratosis may serve as cutaneous markers for internal malignancy especially in unusual presentations, and is also considered as a bad prognostic factor by several authors. In our case, there was no relevant history in regard to malignancy.

Nonsurgical methods such as cryosurgery is commonly performed by dermatologists ${ }^{[2]}$. However, in some cases with large lesions or lesions affecting special areas such as the eyelids, conservative surgical excision and reconstruction are preferred to preserve the functional unit. Invariably, a plastic surgeon's expertise would be needed. As described in the case report, surgical excision was performed with an advancement of the surrounding skin to achieve tension free primary closure without distorting the surrounding structure. This technique utilised the laxity of the elderly's skin surrounding the temple region without distorting the eyelid function. Other methods of reconstruction include skin grafting, but with poor cosmetic outcome or local pivotal flap, using cervico-facial rotational advancement flap in younger patients with less tissue laxity ${ }^{[19]}$.

\section{Conclusion}

Seborrheic keratosis can manifest in an unusual way, and histopathological analysis is warranted to look for concomitant malignancy. Surgical excision with reconstruction is a preferred method at specialised areas in order to preserve aesthetic and functional outcomes.

\section{Conflict of interest}

The author declared no potential conflict of interest with respect to the research, authorship, and/or publication of this article.

\section{References}

1. Hafner C, Vogt T. Seborrheic keratosis. J Dtsch Dermatol Ges 2008; 6(8): 664-677. doi: 10.1111/j.1610-0387.2008. 06788.x.

2. Jackson JM, Alexis A, Berman B, Berson DS, Taylor S, et al. Current understanding of seborrheic keratosis: Prevalence, etiology, clinical presentation, diagnosis, and management. J Drugs Dermatol 2015; 14(10): 1119-1125.

3. Baer RL. Giant pedunculated seborrheic keratosis. Arch Dermatol 1979; 115(5): 627. doi: 10.1001/archderm.1979. 04010050057023.

4. Beheshti A, Hajmanoochehri F. Seborrheic keratosis with Bowenoid transformation. Comp Clin Path 2015; 24(3): 703-704. doi: 10.1007/s00580-014-2042-3.

5. Boyd AS, Su PF, Shyr Y, Tang YW. Squamous cell carcinomas in situ arising in seborrheic keratoses: An association with concomitant immunosuppression? Int J Dermatol 2014; 53(11): 1346-1350. doi: 10.1111/ijd.12086.

6. Gaffney DC, Muir JB, De'Ambrosis B. Malignant change in seborrhoeic keratoses in a region with high solar ultraviolet levels. Australas J Dermatol 2014; 55(2): 142-144. doi: 10.1111/ajd.12035.

7. Terada T. Pigmented Bowen disease arising in pigmented reticulated seborrheic keratosis. Int J Clin Oncol 2010; 
15(6): 608-610. doi: 10.1007/s10147-010-0086-1.

8. Tsai TM, Wu YH, Yang KC, Yang CY, Tsai TH, et al. Sebaceous carcinoma associated with seborrheic keratosis. J Cutan Med Surg 2010; 14(5): 240-244. doi: 10.2310/7750. 2010.09059.

9. Rajabi P, Adibi N, Nematollahi P, Heidarpour M, Eftekhari $\mathrm{M}$, et al. Bowenoid transformation in seborrheic keratosis: A retrospective analysis of 429 patients. J Res Med Sci 2012; 17(3): 217-221.

10. Bandyopadhyay D, Saha A, Mishra V. Giant perigenital seborrheic keratosis. Indian Dermatol Online J 2015; 6(1): 39-41. doi: 10.4103/2229-5178.148939.

11. Longo C, Moscarella E, Piana S, Lallas A, Carrera C, et al. Not all lesions with a verrucous surface are seborrheic keratoses. J Am Acad Dermatol 2014; 70(6): e121-e123. doi: 10.1016/j.jaad.2013.10.042.

12. Thomas I, Kihiczak NI, Rothenberg J, Ahmed S, Schwartz RA. Melanoma within the seborrheic keratosis. Dermatol Surg 2004; 30(4): 559-561. doi: 10.1111/j.1524-4725.2004. 30178.x.

13. Birnie AJ, Varma S. A dermatoscopically diagnosed collision tumour: Malignant melanoma arising within a sebor- rhoeic keratosis. Clin Exp Dermatol 2008; 33(4): 512-513. doi: 10.1111/j.1365-2230.2008.02715.x.

14. Salerni G, Alonso C, Gorosito M, Fernández-Bussy R. Seborrheic keratosis-like melanoma. J Am Acad Dermatol 2015; 72(1 Supp1): S53-S55. doi: 10.1016/j.jaad.2014. 07. 009.

15. Lim C. Seborrhoeic keratoses with associated lesions: A retrospective analysis of 85 lesions. Australas J Dermatol 2006;47(2): 109-113. doi:10.1111/j.1440-0960.2006.00258.x.

16. Vun Y, De'Ambrosis B, Spelman L, Muir JB, Yong- Gee S, et al. Seborrhoeic keratosis and malignancy: Collision tumour or malignant transformation? Australas J Dermatol 2006;47(2):106-108. doi:10.1111/j.1440-0960.2006.00242. x.

17. Noiles K, Vender R. Are all seborrheic keratoses benign? Review of the typical lesion and its variants. J Cutan Med Surg 2008; 12(5): 203-210. doi: 10.2310/7750.2008. 07096.

18. Ponti G, Luppi G, Losi L, Giannetti A, Seidenari S. Leser-Trélat syndrome in patients affected by six multiple metachronous primitive cancers. J Hematol Oncol 2010; 3: 2. doi: 10.1186/1756-8722-3-2.

19. Baker SR. Local flaps in facial reconstruction: Expert consult. $3^{\text {rd }}$ ed. Philadelphia: Elsevier Health Sciences; 2014. 